\title{
New Insights into the Stochastic Geometry Analysis of Dense CSMA Networks
}

\author{
Giusi Alfano \\ Dipartimento di Elettronica \\ Politecnico di Torino, Italy \\ Email: alfano@tlc.polito.it
}

\author{
Michele Garetto \\ Dipartimento di Informatica \\ Università di Torino, Italy \\ Email: garetto@di.unito.it
}

\author{
Emilio Leonardi \\ Dipartimento di Elettronica \\ Politecnico di Torino, Italy \\ Email: leonardi@polito.it
}

\begin{abstract}
Stochastic geometry proves to be a powerful tool for modeling dense wireless networks adopting random MAC protocols such as ALOHA and CSMA. The main strength of this methodology lies in its ability to account for the randomness in the nodes' location jointly with an accurate description at the physical layer, based on the SINR, that allows to consider also random fading on each link. Existing models of CSMA networks adopting the stochastic geometry approach suffer from two important weaknesses: 1) they permit to evaluate only spatial averages of the main performance measures, thus hiding possibly huge discrepancies in the performance achieved by individual nodes; 2) they are analytically tractable only when nodes are distributed over the area according to simple spatial processes (e.g., the Poisson point process). In this paper we show how the stochastic geometry approach can be extended to overcome the above limitations, allowing to obtain node throughput distributions as well as to analyze a significant class of topologies in which nodes are not independently placed.
\end{abstract}

\section{INTRODUCTION}

The last few years have witnessed the proliferation of WiFi Access Points (APs) in many urban areas, both at public places (airports, train stations, coffee shops, university campuses) and private premises (residential homes, corporate buildings). Today, WiFi-enabled home routers are commonly offered upon subscription of a high-speed Internet service (e.g., ADSL or cable). As consequence, at any spot of a large city it is not uncommon to be in the communication range of several APs (even more than ten). The high density of APs can easily lead to poor performance due to increasing levels of interference among APs employing the same channel. The complex behavior of dense networks of interfering APs is still far to be fully understood, making of crucial importance the availability of analytical models that can predict the impact of the cumulative interference produced by the APs operating over the same channel, by incorporating a realistic description at the physical layer.

Traditional models of CSMA (and in particular 802.11) networks are based on Markovian approaches. When all APs are in the sensing range of each other, very accurate and detailed models of 802.11 are available [1]. Large-scale wireless networks employing CSMA are instead very hard to analyze, especially if one wants to incorporate the specific details of 802.11 and its impairments (e.g., hidden terminals) [2]. Simplified versions of CSMA are still amenable to Markovian

This work has been supported by the European Comission through TREND NoE (Towards Real Energy-efficient Network Design). analysis by exploiting the independent sets method originally proposed in [3], which has recently been revisited, coupled with statistical physics arguments, to explain the severe unfairness observed in heavily loaded networks [4]. However, markovian models can only be applied to either small or large but regular topologies, and fail to incorporate physical layer effects (such as cumulative interference, fading etc.).

From a different perspective, stochastic geometry has been proposed as a powerful tool to analyze random, arbitrarily large and arbitrarily dense wireless networks employing variants of ALOHA and CSMA, with an accurate description at the physical layer based on the SINR [5]. However, existing stochastic geometric models of CSMA (see Section II) suffer from two main limitations: i) they assume that APs are distributed according to a Poisson process; ii) they evaluate only spatial performance averages over the entire population of nodes.

While the assumption that nodes are distributed according to a Poisson process can well represent scenarios in which APs independently pop up in an uncoordinated manner (such as residential APs), it is not adequate in the case in which APs are placed in a coordinated/planned manner (e.g., corporate WLANs, or publicly accessible APs deployed by the same Internet Service Provider).

On the other hand, while spatial averages permit to capture within a single metric the average node performance (such as throughput and delay), they completely hide the possibly huge discrepancies in the performance achieved by different nodes. On this regard, [6] has shown (for dense networks employing ALOHA) that the spatial average of the access delay may become unbounded (through a phase transition) even if the spatial average of the throughput is not null. This effect is caused by strong inhomogeneities among nodes' throughputs: a fraction of the nodes in the network are almost starved so that their access delay is extremely large, with a dramatic impact on the overall spatial average.

In this paper we address both limitations above providing two main contributions: first, we show how to extend the stochastic geometry analysis of dense CSMA networks to obtain approximate throughput distributions, instead of just averages; second, we propose an analytically tractable technique that permits to consider scenarios in which APs are not distributed according to a Poisson point process. In particular, our methodology allows to analyze random topologies subject to a minimum separation constraint, which are likely to be 
found in planned networks.

Our work provides new insights into the behavior of random, densely deployed CSMA network, such as: what is the impact of the sensing threshold on the node throughput distribution? What is the impact of physical channel parameters (such as path loss exponent, fading) on nodes throughput (distribution)? Which performance gains are achievable by a more careful strategy of AP placement, with respect to uncoordinated deployment? Such questions have not yet been analytically addressed, to the best of our knowledge, in random CSMA networks.

\section{RELATED WORK}

We limit ourselves to mentioning related works based on stochastic geometry, which has been applied to modeling wireless networks only in the last few years (see [5], [7] for a more comprehensive overview of the literature). While the behavior of ALOHA has been throughly investigated [8], [9], [6], CSMA-like protocols cannot be analyzed exactly due to intrinsic difficulties in characterizing the point process of nodes which are allowed concurrently to transmit. In [10] (which is the starting point of our work), the authors proposed a modified Matérn point process to capture key properties of CSMA networks (for Poisson node distribution) while providing a conservative estimate of the transmitters' density. Less conservative hard-core models, such as the Simple Sequential Inhibition [11], [12], turn out to be very challenging to analyze.

In [7, Ch.3] the authors characterize the interference distribution in Poisson networks, both in the absence and in the presence of fading. For CSMA networks, they assume that the interference is produced only by transmitters located outside a disc of radius equal to the sensing range. They also consider the case in which transmitters are distributed according to a Poisson cluster process. The work [13] investigates the optimization of the sensing range in the absence of fading (to maximize the aggregate capacity under the SINR model) for CSMA-like networks where nodes form a Poisson process. In [14] authors develop simple bounds to the outage probability of unslotted ALOHA and CSMA in Poisson networks.

More recently, it has been found that, in the high Signal-toInterference regime (i.e., when the density of interferers goes to zero), it is possible to characterize the asymptotic behavior of the outage probability and of the transmission capacity for general isotropic distributions of the transmitters, including the (CSMA-like) Matérn hard-core process [15].

\section{NETWORK MODEL}

\section{A. Location of Access Points and users}

We first assume, similarly to previous works [10], that Access Points (APs) are located according to a homogeneous Poisson point process over the plane with intensity $\lambda_{a}$. This assumption is fairly reasonable when APs are deployed in a fully unplanned fashion, such as in residential scenarios. Indeed, the Poisson assumption implies that APs are uniformly and independently located over the area, which reflects the lack of coordination of unplanned deployment. In Section VI we will propose a different model of APs location which we believe to be suitable to represent planned scenarios. We denote by $\Phi_{A}=\left\{\mathbf{x}_{1}, \mathbf{x}_{2}, \ldots \mathbf{x}_{k}, \ldots\right\}$ the set of AP locations.
We assume that users are always associated with their closest AP. In particular, we suppose that, for each AP, the associated users (we assume there is at least one user per AP) are uniformly distributed within the AP's Voronoi cell.

\section{B. Radio propagation model}

We assume that the power received at a point $\mathrm{x}$ from a transmitter located at a point $\mathbf{y}$, denoted by $P(\mathbf{x}, \mathbf{y})$, is given by $P(\mathbf{x}, \mathbf{y})=P \cdot l(\mathbf{x}, \mathbf{y}) \cdot F(\mathbf{x}, \mathbf{y})$, where:

- $P$ is the (fixed) transmitted power, common to all APs;

- $l(\mathbf{x}, \mathbf{y})$ is the deterministic component of the path loss between $\mathbf{x}$ and $\mathbf{y}$;

- $F(\mathbf{x}, \mathbf{y})$ is a random variable representing fading and shadowing on the wireless link between $\mathbf{x}$ and $\mathbf{y}$.

We suppose that $l(\mathbf{x}, \mathbf{y})$ depends only on the Euclidean distance $d(\mathbf{x}, \mathbf{y})$ between the transmitter and the receiver. Even if all our expressions hold for a general function $l(d(\mathbf{x}, \mathbf{y}))$, the results that we will present in this paper are obtained under the following model accounting for near-field effects:

$$
l(\mathbf{x}, \mathbf{y})=\left\{\begin{array}{cl}
d(\mathbf{x}, \mathbf{y})^{-\alpha} & \text { if } d(\mathbf{x}, \mathbf{y})>r_{0} \\
r_{0}^{-\alpha} & \text { if } d(\mathbf{x}, \mathbf{y}) \leq r_{0}
\end{array}\right.
$$

where $r_{0}>0$, and $\alpha>2$ is the path-loss exponent, which depends on the environment. We will also use the equivalent notation $l(r)=\min \left(r, r_{0}\right)^{-\alpha}$ for all positive real numbers $r$. The random variables $F(\mathbf{x}, \mathbf{y})$ are instead assumed to be i.i.d., with generic distribution, for any pair $(\mathbf{x}, \mathbf{y})^{1}$. Let $g_{F}(\zeta)$ be the probability density function of the fading/shadowing random variable, and $\phi_{F}(s)$ its Laplace transform. We denote by $G_{F}(\zeta)$ the cumulative distribution function of $g_{F}(\zeta)$, and by $\bar{G}_{F}(\zeta)=1-G_{F}(\zeta)$ the complementary of $G_{F}(\zeta)$.

\section{MAC contention model}

To determine the subset of APs that transmit simultaneously, we adopt the modified Matérn model proposed in [10], which captures key features of CSMA-like protocols while maintaining analytical tractability. In particular, the model captures the fact that an AP refrains from transmitting when it senses the activity of another AP which has extracted a smaller back-off time. This behavior is modeled in the following way: each point $\mathbf{x}$ of $\Phi_{A}$ is attributed an independent mark $t_{\mathbf{x}}$ uniformly distributed in $[0,1]$, representing the back-off time. The node transmits if it does not sense the activity of any other node having smaller mark, i.e., nodes that have extracted a shorter backoff. The subset $\Phi_{T}$ of APs transmitting concurrently can be formally defined as

$$
\Phi_{T}=\left\{\mathbf{x} \in \Phi_{A}: t_{\mathbf{x}}<t_{\mathbf{y}}, \forall \mathbf{y}: P(\mathbf{x}, \mathbf{y})>\sigma\right\}
$$

Notice that, in the absence of fading, set $\Phi_{T}$ becomes a standard hard-core Matérn process with fixed inhibition radius.

The considered MAC contention model ignores collisions, exponential back-off and history of timers. Moreover, it is more suitable to describe the synchronized, slotted version of CSMA, in which nodes independently extract a back-off time at the beginning of a slot, and all concurrent transmissions finish by the end of the slot. The behavior of unslotted,

\footnotetext{
${ }^{1}$ In the following we will also denote by $F$ the generic marginal random variable $F(\mathbf{x}, \mathbf{y})$.
} 
asynchronous CSMA is much more complex, and tends to introduce severe short- and long-term unfairness among the nodes, especially when the average back-off time is much smaller than the packet duration, as in 802.11 (see [4]). Despite its approximate nature, the modified Matérn process provides a conservative, reasonable estimate of the transmitters' density in 802.11 networks, as shown in [10] by comparison with ns2 simulations.

\section{Transmission model and throughput analysis}

We assume that a transmission is successfully decoded if the SINR at the receiver is larger than a predefined threshold $\beta$, which determines the instantaneous rate. We focus on downlink traffic only (i.e., from the APs to the users), assuming that uplink traffic is negligible. Moreover, we assume that APs are constantly backlogged by packets to send. A user located at $\mathbf{x}$ correctly receives data sent by its closest AP at $\mathbf{y}$ when

$$
\operatorname{SINR}(\mathbf{x})=\frac{\mathrm{P}(\mathbf{x}, \mathbf{y})}{\mathrm{N}_{0}+\sum_{\mathbf{j} \in \Phi_{\mathrm{T}} \backslash \mathbf{y}} \mathrm{P}(\mathbf{x}, \mathbf{j})}>\beta
$$

where $N_{0}$ is the (constant) ambient noise power and $I(\mathbf{x}, \mathbf{y})=$ $\sum_{\mathbf{j} \in \Phi_{A} \backslash \mathbf{y}} P(\mathbf{x}, \mathbf{j})$ is the cumulative interference produced by all other transmitting APs.

In our throughput analysis we focus on a typical AP and consider the instantaneous rate at which this AP is transmitting at a given time to one of its associated users (there exists by hypothesis at least one user for each AP), which are assumed to be granted an equal number of transmission opportunities irrespective of their distance from the AP. Notice that we are not computing the user-perceived throughput but the aggregate throughput of the AP, to avoid the additional complexity of analyzing the bandwidth sharing among users associated to the same AP (i.e., located in the same Voronoi cell). The user throughput can be derived from the AP throughput adopting the approach proposed in [10].

For simplicity, we consider a unique threshold $\beta$, and we normalize to one the corresponding transmission rate. It should be clear, however, that if we are able to evaluate the probability of (3) for an arbitrary $\beta$, than we can easily compute the throughput achievable with a set of different rates (modulation schemes) selected by an auto-rate function of the SINR (for example, a piecewise constant function).

Under our assumptions, the instantaneous throughput of the typical AP equals the product of probabilities that: i) the AP is transmitting; ii) the transmission is successfully decoded by the intended receiver. In this paper we are interested both in the spatial average of the AP throughput and in its spatial distribution.

\section{BASELINE ANALYSIS}

In this section we briefly recall the technique proposed in [10] to approximate the spatial average $\mathcal{T}$ of the AP throughput in the case of a Poisson distribution of APs, to put the reader in a position to understand our extended analysis. The spatial average of the throughput can be obtained by first conditioning on the distance $r$ between the $\mathrm{AP}$ and the user:

$$
\mathcal{T}=\int_{0}^{\infty} \mathcal{T}(r) f_{D}(r) \mathrm{d} r
$$

where $f_{D}(r)$ is the probability density of the distance between a user and its closest AP. In the Poisson case,

$$
f_{D}(r, \text { Poisson })=2 \pi r \lambda_{a} e^{-\lambda_{a} \pi r^{2}}
$$

The conditional throughput $\mathcal{T}(r)$ can be expressed as the product of: the conditional transmission probability $p_{T}(r)$ of the tagged AP, given that there is a user at distance $r$ whose closest AP is the tagged AP; ii) the probability $p_{s}(r)$ of successful reception at distance $r$ from the AP, given the occurrence of the previous event.

To compute the exact value of $p_{T}(r)$, suppose that the receiving node is located at point $\mathbf{y}=(r, 0)$, and its closest $\mathrm{AP}$ is located at the origin $\mathbf{0}$ (we denote this AP with $\mathbf{0}$ in the following). We have

$p_{T}(r)=\int_{0}^{1} e^{-\lambda_{a} t_{\mathbf{0}} \int_{\mathbb{R}^{2} \backslash \mathcal{B}(\mathbf{y}, r)} S(\mathbf{x}) \mathrm{d} \mathbf{x}} \mathrm{d} t_{\mathbf{0}}=\frac{1-e^{-\lambda_{a} \int_{\mathbb{R}^{2} \backslash \mathcal{B}(\mathbf{y}, r)} S(\mathbf{x}) \mathrm{d} \mathbf{x}}}{\lambda_{a} \int_{\mathbb{R}^{2} \backslash \mathcal{B}(\mathbf{y}, r)} S(\mathbf{x}) \mathrm{d} \mathbf{x}}$

where $\mathcal{B}(\mathbf{y}, r)$ is the ball of radius $r$ centered at $\mathbf{y}$, whereas $S(\mathbf{x})$ is the probability for $\mathbf{0}$ to sense another AP located at $\mathbf{x}$. The spatial integral in (6) can be intuitively explained (see [5] for a rigorous proof based on Palm probability and Slivnyak's Theorem) considering that the infinitesimal area $\mathrm{d} x$ centered at any point $\mathbf{x}$ of the plane (excluding ball $\mathcal{B}(\mathbf{y}, r)$, which by hypothesis does not contain APs) must be free of nodes sensed by $\mathbf{0}$. Conditioning on the mark $t_{\mathbf{0}}$ of AP $\mathbf{0}$ (which provides the outer integral in (6)), the intensity of the Poisson Point process of APs that can potentially prevent $\mathbf{0}$ from transmitting, i.e. those having mark smaller than $t_{\mathbf{0}}$, is $\lambda_{a} t_{\mathbf{0}}$. This point process is then further thinned by the (location-dependent) probability $S(\mathbf{x})$ that $\mathbf{0}$ indeed senses an AP located at $\mathbf{x}$. Under the modified Matérn model of CSMA, all nodes with mark smaller than $t_{0}$ are assumed to transmit with probability one, hence $S(\mathbf{x})$ equals $\operatorname{Pr}[P(\mathbf{0}, \mathbf{x})>\sigma]=\bar{G}_{F}\left(\frac{\sigma}{P l(x)}\right)$, (where $x$ denotes the euclidean norm $\|\mathbf{x}\|)$ which is the probability that the signal transmitted at $\mathbf{x}$ is received by $\mathbf{0}$ with power above the sensing threshold $\sigma$.

To evaluate the probability of successful reception $p_{s}(r)$, we need to compute (though in an approximate way) the cumulative interference produced by all of the other APs concurrently transmitting with $\mathbf{0 .}$.

For this, we first need an auxiliary function $h\left(x, \lambda_{a}\right)$, which provides the probability that an AP transmits, conditioned on the fact that there is a transmitting AP at distance $x$ from it, belonging to the same Poisson point process of intensity $\lambda_{a}$. An exact evaluation of $h\left(x, \lambda_{a}\right)$ can be carried out as follows. Let $\mathbf{0}$ and $\mathbf{x}$ be two APs at distance $x$ from each other, and let $t_{\mathbf{0}}$ and $t_{\mathbf{x}}$ be their marks. We can express $h\left(x, \lambda_{a}\right)$ as

$$
\begin{aligned}
h\left(x, \lambda_{a}\right)=\mathbb{P}_{\Phi_{A}}^{\mathbf{0 ,}, \mathbf{x}}\left\{\mathbf{x} \in \Phi_{T} \mid \mathbf{0} \in\right. & \left.\Phi_{T}\right\}= \\
& \frac{\mathbb{P}_{\Phi_{A}}^{\mathbf{0 , x}}\left\{\mathbf{x} \in \Phi_{T}, \mathbf{0} \in \Phi_{T}\right\}}{\mathbb{P}_{\Phi_{A}}^{\mathbf{0}, \mathbf{x}}\left\{\mathbf{0} \in \Phi_{T}\right\}}
\end{aligned}
$$

where $\mathbb{P}_{\Phi_{A}}^{\mathbf{0 , x}}\{\}$ is the probability law associated to the point process $\Phi_{A}+\mathbf{0}+\mathbf{x}$; observe that $\mathbb{P}_{\Phi_{A}}^{\mathbf{0}, \mathbf{x}}\left\{\mathbf{x} \in \Phi_{T}, \mathbf{0} \in \Phi_{T}\right\}$ is the probability that two APs placed at distance $x$ from each 
other transmit concurrently, whereas $\mathbb{P}_{\Phi_{A}}^{\mathbf{0}, \mathbf{x}}\left\{\mathbf{0} \in \Phi_{T}\right\}$ is the probability that $\mathbf{0}$ transmits, given that there is another AP at distance $x$ from it (not necessarily concurrently transmitting).

We start computing the joint probability that $\mathbf{0}$ and $\mathbf{x}$ transmits concurrently. When considering all possible combinations of $t_{\mathbf{x}}$ and $t_{\mathbf{0}}$, we can limit ourselves to the case $t_{\mathbf{0}}<t_{\mathbf{x}}$, and then multiply the result by two (the case $t_{\mathbf{x}}<t_{\mathbf{0}}$ is symmetric). Besides nodes $\mathbf{0}$ and $\mathbf{x}$, we need to consider the superposition of two independent homogeneous Poisson point processes: a process of intensity $\lambda_{a} t_{\mathbf{0}}$, related to those nodes having mark $t<t_{\mathbf{0}}$ that can be sensed by nodes $\mathbf{0}$ and/or node $\mathbf{x}$; a process of intensity $\lambda\left(t_{\mathbf{x}}-t_{\mathbf{0}}\right)$, related to those nodes having mark $t_{\mathbf{0}}<t<t_{\mathbf{x}}$ that can be sensed by node $\mathbf{x}$ only. Hence we can express $\mathbb{P}_{\Phi_{A}}^{\mathbf{0}, \mathbf{x}}\left\{\mathbf{x} \in \Phi_{T}, \mathbf{0} \in \Phi_{T}\right\}$ as

$$
\begin{array}{r}
\mathbb{P}_{\Phi_{A}}^{\mathbf{0}, \mathbf{x}}\left\{\mathbf{x} \in \Phi_{T}, \mathbf{0} \in \Phi_{T}\right\}=2 G_{F}\left(\frac{\sigma}{P l(x)}\right) \\
\int_{0}^{1} \int_{t_{\mathbf{0}}}^{1} e^{-\lambda_{a}\left(t_{\mathbf{x}}-t_{\mathbf{0}}\right) \int_{\mathbb{R}^{2}} S_{\mathbf{x}}(\mathbf{z}) \mathrm{d} \mathbf{z}} \mathrm{d} t_{\mathbf{x}} \\
e^{-\lambda_{a} t_{\mathbf{0}} \int_{\mathbb{R}^{2}} S_{\mathbf{O} \text { or } \mathbf{x}}(\mathbf{z}) \mathrm{d} \mathbf{z}} \mathrm{d} t_{\mathbf{0}}
\end{array}
$$

In the above equation, the term $G_{F}\left(\frac{\sigma}{P l(x)}\right)$ corresponds to the probability that the AP transmitting first is not sensed by the other, which is the only requirement to have the two nodes concurrently transmitting when there are no other nodes in the network; $S_{\mathbf{x}}(\mathbf{z})$ is the probability that node $\mathbf{x}$ senses a node at $\mathbf{z} ; S_{\mathbf{0} \text { or } \mathbf{x}}(\mathbf{z})=1-\left(1-S_{\mathbf{0}}(\mathbf{z})\right)\left(1-S_{\mathbf{x}}(\mathbf{z})\right)$ is the probability that at least one node (between $\mathbf{0}$ and $\mathbf{x}$ ) senses a node at $\mathbf{z}$.

The conditional probability $\mathbb{P}_{\Phi_{A}}^{\mathbf{0}, \mathbf{x}}\left\{\mathbf{0} \in \Phi_{A}\right\}$ can be obtained in a similar way, this time considering all possible combinations of $t_{\mathbf{0}}$ and $t_{\mathbf{x}}$ :

$$
\begin{array}{r}
\mathbb{P}_{\Phi_{A}}^{\mathbf{0}, \mathbf{x}}\left\{\mathbf{0} \in \Phi_{T}\right\}=\int_{0}^{1}\left\{\int_{0}^{t_{\mathbf{x}}} e^{-\lambda_{a} t_{\mathbf{o}} \int_{\mathbb{R}^{2}} S_{\mathbf{0}}(\mathbf{z}) \mathrm{d} \mathbf{z}} \mathrm{d} t_{\mathbf{0}}+\right. \\
\left.\int_{t_{\mathbf{x}}}^{1} G_{F}\left(\frac{\sigma}{P l(x)}\right) e^{-\lambda_{a} t_{\mathbf{0}} \int_{\mathbb{R}^{2}} S_{\mathbf{0}}(\mathbf{z}) \mathrm{d} \mathbf{z}} \mathrm{d} t_{\mathbf{0}}\right\} \mathrm{d} t_{\mathbf{x}}
\end{array}
$$

In the above expression, $G_{F}\left(\frac{\sigma}{P l(x)}\right)$ is the probability that $\mathbf{0}$ does not sense the transmission of $\mathbf{x}$, when $t_{\mathbf{0}}>t_{\mathbf{x}}$.

Having computed $h\left(x, \lambda_{a}\right)$, we can evaluate in an approximate way the cumulative interference plus noise suffered at the receiving node. The approximation consists in assuming that interfering APs form an (in-homogenous) Poisson point process whose local intensity depends (through function $\left.h\left(x, \lambda_{a}\right)\right)$ only on the distance $x$ from the AP transmitting the useful signal. Under this approximation, the Laplace transform $\psi_{I+N_{0}}(s)$ of interference plus noise is given by (see [5] for details),

$$
\begin{aligned}
& \psi_{I+N}(s)=\psi_{I}(s) \psi_{N}(s) \approx \\
& \quad \approx e^{-\lambda_{a} \int_{0}^{2 \pi} \int_{r}^{\infty} h\left(b(\rho, \theta), \lambda_{a}\right)\left[1-\phi_{F}(s P l(\rho))\right] \rho \mathrm{d} \rho \mathrm{d} \theta} e^{-s N_{0}}
\end{aligned}
$$

where the spatial integral is computed using polar coordinates centered at the receiving node. In this coordinate system, $b(\rho, \theta)=\rho^{2}+r^{2}-r \rho \cos (\theta)$ provides the distance of the generic point $(\rho, \theta)$ from the AP transmitting the useful signal, which is assumed to be located at $(r, 0)$.

Recall that the signal is decoded successfully if the received power exceeds threshold $\beta$, see (3). This requires that, for a given value $\xi$ of $\left(I+N_{0}\right)$, the fading variable $F$ is larger than $\frac{\beta \xi}{P l(r)}$. For a general fading distribution, we have

$$
p_{s}(r)=\left.\int_{0}^{\infty} \bar{G}_{F}\left(\frac{\beta \xi}{P l(r)}\right) \mathcal{L}^{-1}\left\{\psi_{I+N_{0}}(s)\right\}\right|_{\xi} \mathrm{d} \xi
$$

which requires to numerically invert the Laplace transform $\psi_{I+N_{0}}(s)$. A direct computation of $p_{s}(r)$ is possible in the special case in which the fading is exponentially distributed (i.e., Rayleigh fading). Indeed, when $F$ is exponential with mean $1 / \mu$, we have

$$
p_{s}(r)=\int_{0}^{\infty} e^{-\frac{\mu \beta \xi}{P l(r)}} \mathrm{d} \operatorname{Pr}\left(I+N_{0} \leq \xi\right)
$$

which is equivalent to evaluate the Laplace transform $\psi_{I+N_{0}}(s)$ at $s=\mu \beta /(P l(r))$. The expression (12) can be generalized to the case in which fading is phase-type distributed, $G_{F}(z)=1-\sum_{i} c_{i}\left(\sum_{k=0}^{K_{i}} \frac{\left(\mu_{i} z\right)^{k}}{k !} e^{-\mu_{i} z}\right)$, with $c_{i} \geq 0$ and $\sum_{i} c_{i}=1$, obtaining:

$$
p_{s}(r)=\sum_{i} c_{i}\left(\sum_{k=0}^{K_{i}} \int_{0}^{\infty} \frac{\gamma_{i}^{k}}{k !} e^{-\gamma_{i}} \mathrm{~d} \operatorname{Pr}\left(I+N_{0} \leq \xi\right)\right)
$$

where $\gamma_{i}=\mu_{i} \beta \xi /(P \ell(r))$. The computation of (13) reduces to evaluate a linear combination of the Laplace transform $\psi_{I+N_{0}}(s)$ and its derivatives at points $s_{i}=\mu_{i} \beta /(P l(r))$.

\section{BEYOND SPATIAL AVERAGES}

In this section we describe how the stochastic geometry approach can be extended to obtain, besides spatial averages, also an estimate of the distribution of the throughput achieved by the APs. Doing so, we will get interesting insights into the impact of several system parameters (especially the sensing threshold and the distribution of fading/shadowing) on the discrepancies that we can observe among the throughputs of different APs in a random network. This analysis is especially important to understand how the natural throughput unbalance due to the randomness in the topology (i.e., the presence of areas more or less populated by nodes) is perturbed by the randomness in the wireless channel, and how this natural unbalance can be mitigated by a proper selection of protocol parameters. We will see that stochastic geometry can provide an answer to these questions in a simple and natural way.

Notice that (4) already contains one source of variability, i.e., the one due to the variable distance between the AP and the receiver, that we can already isolate with the previous analysis. Besides the impact of the distance distribution, we (separately) capture two additional sources of variability: the variability in the transmission probability $p_{T}(r)$, in Section $\mathrm{V}-\mathrm{A}$, and the variability in the probability $p_{s}(r)$ of successful reception, in Section V-B. Then in Section V-C we combine everything together obtaining our final estimate of the throughput distribution. 


\section{A. Variability in the transmission probability}

Clearly, the chance of an AP to access the channel is strongly affected by the pattern of contenders in its neighborhood: even in the presence of fading, the nodes that are most likely to prevent an AP from transmitting are those located in close proximity to it. To capture this fact, we will evaluate the conditional probability $p_{T}^{d}(r, n)$ that the tagged AP, located at $\mathbf{0}$, is allowed to transmit, given that there are $n$ other APs within distance $d$ from it (excluding the empty disc of radius $r$ centered at the receiver), where $d$ is a parameter of the analysis. We have,

$$
p_{T}^{d}(r, n)=\int_{0}^{1} e^{-t_{\mathbf{0}} \mathcal{E}(r, d)}\left(1-t_{\mathbf{0}} \mathcal{I}(r, d)\right)^{n} \mathrm{~d} t_{\mathbf{0}}
$$

where,

$$
\begin{aligned}
\mathcal{E}(r, d) & =\lambda_{a} \int_{\mathbb{R}^{2} \backslash(\mathcal{B}(\mathbf{0}, d) \cup \mathcal{B}(\mathbf{y}, r))} S(\mathbf{x}) \mathrm{d} \mathbf{x} \\
\mathcal{I}(r, d) & =\frac{\int_{\mathcal{B}(\mathbf{0}, d) \backslash \mathcal{B}(\mathbf{y}, r)} S(\mathbf{x}) \mathrm{d} \mathbf{x}}{\int_{\mathcal{B}(\mathbf{0}, d) \backslash \mathcal{B}(\mathbf{y}, r)} \mathrm{d} \mathbf{x}}
\end{aligned}
$$

and $S(\mathbf{x})=\bar{G}_{F}\left(\frac{\sigma}{P l(x)}\right)$.

Proof: Similarly to (6), we condition on the mark $t_{\mathbf{0}}$ associated to AP 0. To transmit, the AP must not sense any other AP having smaller mark. The APs located outside the ball $\mathcal{B}(\mathbf{0}, d)$, having mark smaller than $t_{\mathbf{0}}$, form a Poisson process of intensity $\lambda_{a} t_{0}$ and can be treated exactly in the same way as before, providing the term $e^{-\lambda_{a} t_{0} \mathcal{E}(r, d)}$, which is identical to (6) except for a difference in the spatial integration domain (now we have to exclude two discs from $\mathbb{R}^{2}$ ). Then we need to consider the $n$ APs located in the region $\mathcal{B}(\mathbf{0}, d) \backslash \mathcal{B}(\mathbf{y}, r)$, considering that each of them is uniformly distributed in this region, and with probability $t_{\mathbf{0}}$ it has mark smaller than $t_{\mathbf{0}}$. Quantity $\mathcal{I}(r, d)$ provides the average probability that the AP senses one of them, considering all possible locations within the region $\mathcal{B}(\mathbf{0}, d) \backslash \mathcal{B}(\mathbf{y}, r)$. Since the AP must not sense any of them, we obtain for this set of nodes the term $\left(1-t_{\mathbf{0}} \mathcal{I}(r, d)\right)^{n}$ in (14).

The parameter $d$ has to be chosen with care: if it is too small, the expected number of APs in $\mathcal{B}(\mathbf{0}, d)$ is also small, and the conditioning becomes ineffective. If it is set large, we lose control on the number of critical APs (i.e., the nodes most likely to be sensed). A natural choice is to set $d$ equal to the 'effective' sensing range $R_{0}=l^{-1}(\sigma /(P \bar{F}))$, which is the fixed inhibition radius of a system in which the fading variable is deterministically equal to its mean.

\section{B. Variability in the probability of successful reception}

The probability of successful reception $p_{s}(r)$ at distance $r$ from the AP is strongly affected by the pattern of interferers around the receiver, as well as on the characteristics of the wireless channel (i.e., path loss exponent, and fading/shadowing distribution). To capture this fact, we proceed in a similar way as for the transmission probability, evaluating the conditional probability $p_{s}^{e}(r, m)$ of successful reception, given that there are $m$ APs within distance $e$ from the receiver, with $e>r$ (by construction there are no APs at distance smaller than $r$ ).

The Laplace transform on the corresponding conditional interference plus noise distribution is given by

$$
\begin{gathered}
\psi_{I+N_{0}}^{e}[r, m](s)=e^{-\lambda_{a} \int_{0}^{2 \pi} \int_{e}^{\infty} h\left(b(\rho, \theta), \lambda_{a}\right)\left[1-\phi_{F}(s P l(\rho))\right] \rho \mathrm{d} \rho \mathrm{d} \theta} \\
\left(\frac{\int_{0}^{2 \pi} \int_{r}^{e} h\left(b(\rho, \theta), \lambda_{a}\right) \phi_{F}(s P l(\rho)) \rho \mathrm{d} \rho \mathrm{d} \theta}{\int_{r}^{e} h\left(b(\rho, \theta), \lambda_{a}\right) \rho \mathrm{d} \rho \mathrm{d} \theta}\right)^{m} e^{-s N_{0}}
\end{gathered}
$$

Proof: By definition the cumulative interference at the receiver is the sum of all powers received from transmitting APs other than the good one. The corresponding Laplace transform is the product of the Laplace transform of the individual contributions. The contribution of APs located outside the disc of radius $e$ centered at the receiver can be treated exactly in the same way as before, providing the first exponential term in (15), which is identical to (10) except for the different integration domain. Note that we are using polar coordinates centered at the receiver, with the transmitter located at $(r, 0)$.

Then we need to consider the $m$ APs located in the region $\mathcal{B}(\mathbf{0}, e) \backslash \mathcal{B}(\mathbf{0}, r)$, considering that each of them is located at point $(\rho, \theta)$ with density proportional to the local intensity $\lambda_{a} h\left(b(\rho, \theta), \lambda_{a}\right)$ of the inhomogeneous Poisson process of APs concurrently transmitting. Hence the contribution of each of the $m$ APs is

$$
\frac{\int_{0}^{2 \pi} \int_{r}^{e} h\left(b(\rho, \theta), \lambda_{a}\right) \phi_{F}(s P l(\rho)) \rho \mathrm{d} \rho \mathrm{d} \theta}{\int_{r}^{e} h\left(b(\rho, \theta), \lambda_{a}\right) \rho \mathrm{d} \rho \mathrm{d} \theta}
$$

Considering also the contribution of the ambient noise, we get the expression in (15).

The conditional probability $p_{s}^{e}(r, m)$ can then be derived from $\psi_{I+N_{0}}^{e}[r, m](s)$ according to (11), (12), (13), respectively for general, Rayleigh or phase type fading distribution. Again, parameter $e$ has to be chosen with care, trying to isolate those APs which are responsible for the highest variability in the interference. In our results we have set $e=1.5 r$, which has empirically been found to provide the best fit with simulations.

\section{Throughput distribution}

Putting things together, we define the conditional throughput $\mathcal{T}(n, m, r)$ of an AP, located at $\mathbf{r}$, transmitting to a user located at the origin $\mathbf{0}$, given that there are $n$ other APs within the area $\mathcal{B}(\mathbf{r}, d) \backslash \mathcal{B}(\mathbf{0}, r)$ and $m$ other APs in the ring $\mathcal{B}(\mathbf{0}, e) \backslash \mathcal{B}(\mathbf{0}, r)$ :

$$
\mathcal{T}(r, n, m)=p_{T}^{d}(r, n) p_{s}^{e}(r, m)
$$

Denoting by $\mathcal{A}_{r}^{d}$ the area of region $\mathcal{B}(\mathbf{r}, d) \backslash \mathcal{B}(\mathbf{0}, r)$, the probability $F_{r}^{d}(n)$ to find $n$ other APs in it is

$$
F_{r}^{d}(n)=e^{-\lambda_{a} \mathcal{A}_{r}^{d}} \frac{\left(\lambda_{a} \mathcal{A}_{r}^{d}\right)^{n}}{n !}
$$

Instead, the probability $F_{r}^{e}(n)$ to find $m$ other APs in the ring $\mathcal{B}(\mathbf{0}, e) \backslash \mathcal{B}(\mathbf{0}, r)$ is

$$
\begin{array}{r}
F_{r}^{e}(n) \approx e^{-\lambda_{a} \int_{0}^{2 \pi} \int_{r}^{e} h\left(b(\rho, \theta), \lambda_{a}\right) \rho \mathrm{d} \rho \mathrm{d} \theta} \\
\frac{\left(\lambda_{a} \int_{0}^{2 \pi} \int_{r}^{e} h\left(b(\rho, \theta), \lambda_{a}\right) \rho \mathrm{d} \rho \mathrm{d} \theta\right)^{m}}{m !}
\end{array}
$$


The joint probability $F_{r}^{d, e}(n, m)$ to find $n$ APs in region $\mathcal{B}(\mathbf{r}, d) \backslash \mathcal{B}(\mathbf{0}, r)$ and $m$ APs in region $\mathcal{B}(\mathbf{0}, e) \backslash \mathcal{B}(\mathbf{0}, r)$ can be approximated as

$$
F_{r}^{d, e}(n, m) \approx F_{r}^{d}(n) F_{r}^{e}(n)
$$

where the approximation lies in the fact that the above two regions are non disjoint, thus the numbers of points falling in them are not independent. At last, we can express our estimate of the throughput distribution according to:

$$
\mathbb{P}\{\mathcal{T}<\eta\} \approx \sum_{n} \sum_{m} \int_{0}^{\infty} \mathbf{1}_{(\mathcal{T}(r, n, m)<\eta)} F_{r}^{d, e}(n, m) f_{D}(r) \mathrm{d} r
$$

\section{BEYOND THE POISSON PROCESS}

Usually in planned networks APs are not placed independently of each other. Hence the Poisson point process is not well suited to describe controlled network topologies. In particular, in the Poisson point process we can find nodes arbitrarily close to each other, which is something that any reasonable strategy of nodes placement tries to avoid. To minimize mutual interference, while maximizing area coverage, the optimal solution would be to place the APs according to a regular tessellation of the plane (e.g., a triangular lattice). However, APs cannot in general be deployed at any location, due to physical and cost constraints. Hence some randomness in the AP topology has to be considered. To reflect the above facts, we model the point process of APs by a hardcore Matérn process of parameters $(\lambda, R)$, which guarantees a minimum separation constraint of $R$ between APs. Notice that this model can also be used to represent, in unplanned scenarios, the repulsive effect induced by intelligent channel selection schemes, or, in the context of green networking, the effect of switching off redundant APs covering the same region of the network area. Fig. 1 shows a portion of a sample topology generated by a Matern process with parameters $\lambda=10 / \pi, R=1$.

In this section we will show that the Matérn model of AP placement can be smoothly incorporated in the previous analysis, resorting to the same Poisson approximation of the (conditional) Matérn process.

\section{A. Transmission probability}

Similarly to what has been done before to characterize the set of transmitting APs, we first characterize the set of AP locations by computing the probability $g(x, \lambda, R)$ that, given the existence of an AP in the origin, we find another AP at distance $x$ from it. More formally, let $\Phi$ be the set of 'candidate' AP locations generated by the original Poisson point process of rate $\lambda$, and let $\mathbb{P}_{\Phi}^{\mathbf{0}, \mathbf{x}}\{\}$ be the probability law associated with point process $\Phi+\mathbf{0}+\mathbf{x}$. The desired function

$$
g(x, \lambda, R)=\mathbb{P}_{\Phi}^{\mathbf{0}, \mathbf{x}}\left\{\mathbf{x} \in \Phi_{A} \mid \mathbf{0} \in \Phi_{A}\right\}
$$

can be evaluated in a way analogous to function $h\left(x, \lambda_{a}\right)$ in (7), although with a very different meaning (here we are characterizing the set of APs location, not yet the set of transmitting APs). In particular, by specializing (7) to the case

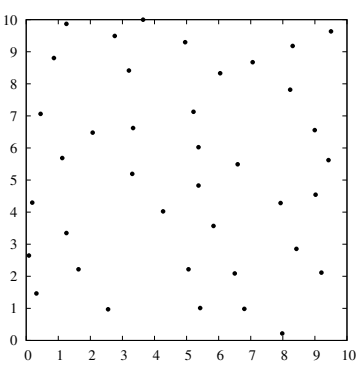

Fig. 1. Example of topology generated by a Matérn process with $\lambda=10 / \pi, R=1$.

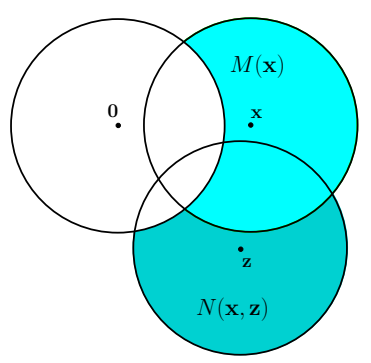

Fig. 2. Geometry used to compute joint/conditional probabilities of node patterns in the Matérn process. of no fading (we consider now a hard-core Matérn process), we obtain the closed form expression

$$
\begin{aligned}
g(x, \lambda, R) & =\mathbf{1}(x>R) \frac{2}{1-e^{-\lambda \pi R^{2}}} \\
& {\left[\frac{1-e^{-\lambda\left(\pi R^{2}+M(x)\right)}}{\lambda\left(\pi R^{2}+M(x)\right)}-e^{-\lambda \pi R^{2}} \frac{1-e^{-\lambda M(x)}}{\lambda M(x)}\right] }
\end{aligned}
$$

where $M(x)$ is the area of $\mathcal{B}(\mathbf{x}, R) \backslash \mathcal{B}(\mathbf{0}, R)$, illustrated in Figure 2 by a light gray region. Then we approximate the law $\lambda_{a}^{0}(\mathbf{x})$ of $\Phi_{A}$, conditioned on the event $\mathbf{0} \in \Phi_{A}$, by an in-homogeneous Poisson point process of intensity

$$
\lambda_{a}^{0}(\mathbf{x})=\lambda g(x, \lambda, R)
$$

At last, the conditional transmission probability $p_{T}(r)$ can be easily obtained extending (6) to the case in which APs are placed according to an inhomogeneous Poisson process:

$$
p_{T}(r)=\int_{0}^{1} e^{-t_{\mathbf{0}} \int_{\mathbb{R}^{2} \backslash \mathcal{B}(\mathbf{y}, r)} \lambda_{a}^{0}(\mathbf{x}) S(\mathbf{x}) \mathrm{d} \mathbf{x}} \mathrm{d} t_{\mathbf{0}}
$$

\section{B. Probability of successful reception}

To evaluate the probability of successful reception $p_{s}(r)$, we need to characterize the set of APs transmitting concurrently with the tagged AP located at $\mathbf{0}$. The key step consists again in computing function $h(x, \lambda, R)=\mathbb{P}_{\Phi_{A}}^{\mathbf{0}, \mathbf{x}}\left\{\mathbf{x} \in \Phi_{T} \mid \mathbf{0} \in \Phi_{T}\right\}$, which can be interpreted as the conditional probability that AP $\mathrm{x} \in \Phi_{A}$ transmits, given that AP $\mathbf{0} \in \Phi_{A}$ is transmitting. Function $h(x, \lambda, R)$ can then be used to characterize (in an approximate way) the law of transmitting APs around AP $\mathbf{0 .}$

The computation of $h(x, \lambda, R)$ can be carried out by approximating the law of the other APs (different from $\mathbf{x}$ and $\mathbf{0}$ ) with an inhomogeneous Poisson process of intensity $\lambda_{a}^{\mathbf{0}, \mathbf{x}}(\mathbf{z})=$ $\lambda \mathbb{P}_{\Phi}^{\mathbf{0}, \mathbf{x}, \mathbf{z}}\left\{\mathbf{z} \in \Phi_{A} \mid \mathbf{0} \in \Phi_{A}, \mathbf{x} \in \Phi_{A}\right\}$ where $\mathbb{P}_{\Phi}^{\mathbf{0}, \mathbf{x}, \mathbf{z}}\{\}$ is the probability law associated to the point process $\Phi+\mathbf{0}+\mathbf{x}+\mathbf{z}$. Function $h(x, \lambda, R)$ can then be computed by extending (8) and (9) to the case of an in-homogeneous Poisson process of intensity $\lambda_{a}^{\mathbf{0}, \mathbf{x}}(\mathbf{z})$. In particular, (8) becomes:

$$
\begin{array}{r}
\mathbb{P}_{\Phi_{A}}^{\mathbf{0}, \mathbf{x}}\left\{\mathbf{x} \in \Phi_{T}, \mathbf{0} \in \Phi_{T}\right\}=2 G_{F}\left(\frac{\sigma}{P l(x)}\right) \\
\int_{0}^{1} \int_{t_{\mathbf{0}}}^{1} e^{-\left(t_{\mathbf{x}}-t_{\mathbf{0}}\right) \int_{\mathbb{R}^{2}} \lambda_{a}^{\mathbf{0}, \mathbf{x}}(\mathbf{z}) S_{\mathbf{x}}(\mathbf{z}) \mathrm{d} \mathbf{z}} \mathrm{d} t_{\mathbf{x}} \\
e^{-t_{\mathbf{0}} \int_{\mathbb{R}^{2}} \lambda_{a}^{\mathbf{0}, \mathbf{x}}(\mathbf{z}) S_{\mathbf{0} \text { or } \mathbf{x}}(\mathbf{z}) \mathrm{d} \mathbf{z}} \mathrm{d} t_{\mathbf{0}}
\end{array}
$$


and similarly for (9).

To compute $\lambda_{a}^{\mathbf{0}, \mathbf{x}}(\mathbf{z})$, we extend the approach used to derive (17) to the case of three points, obtaining

$$
\begin{gathered}
\lambda_{a}^{\mathbf{0 , x}}(\mathbf{z})=\lambda \mathbb{P}_{\Phi}^{\mathbf{0}, \mathbf{x}, \mathbf{z}}\left\{\mathbf{z} \in \Phi_{A} \mid \mathbf{0} \in \Phi_{A}, \mathbf{x} \in \Phi_{A}\right\}= \\
=\lambda \frac{\mathbb{P}_{\Phi}^{\mathbf{0 , x}, \mathbf{z}}\left\{\mathbf{z} \in \Phi_{A}, \mathbf{0} \in \Phi_{A}, \mathbf{x} \in \Phi_{A}\right\}}{\mathbb{P}_{\Phi}^{\mathbf{0}, \mathbf{x}, \mathbf{z}}\left\{\mathbf{0} \in \Phi_{A}, \mathbf{x} \in \Phi_{A}\right\}}= \\
=\lambda \frac{6 \mathbb{P}_{\Phi}^{\mathbf{0}, \mathbf{x}, \mathbf{z}}\left\{\mathbf{z} \in \Phi_{A}, \mathbf{0} \in \Phi_{A}, \mathbf{x} \in \Phi_{A} \mid t_{\mathbf{z}}<t_{\mathbf{x}}<t_{\mathbf{0}}\right\}}{2 \mathbb{P}_{\Phi}^{\mathbf{0}, \mathbf{x}, \mathbf{z}}\left\{\mathbf{0} \in \Phi_{A}, \mathbf{x} \in \Phi_{A} \mid t_{\mathbf{x}}<t_{\mathbf{0}}\right\}}= \\
=3 \lambda \frac{\int_{0}^{1} \int_{t_{\mathbf{z}}}^{1} \int_{t_{\mathbf{x}}}^{1} e^{-\lambda t_{\mathbf{0}} \pi R^{2}} e^{-\lambda t_{\mathbf{x}} M(\mathbf{x})} e^{-\lambda t_{\mathbf{z}} N(\mathbf{x}, \mathbf{z})} \mathrm{d} t_{\mathbf{0}} \mathrm{d} t_{\mathbf{x}} \mathrm{d} t_{\mathbf{z}}}{\int_{0}^{1} \int_{t_{\mathbf{x}}}^{1} e^{-\lambda t_{0} \pi R^{2}} e^{-\lambda t_{\mathbf{x}} M(\mathbf{x})} \mathrm{d} t_{\mathbf{0}} \mathrm{d} t_{\mathbf{x}}}=
\end{gathered}
$$

which can be computed in closed form as function of the area $M(\mathbf{x})$ of $\mathcal{B}(\mathbf{x}, R) \backslash \mathcal{B}(\mathbf{0}, R)$, and of the area $N(\mathbf{x}, \mathbf{z})$ of $\mathcal{B}(\mathbf{z}, R) \backslash(\mathcal{B}(\mathbf{x}, R) \cup \mathcal{B}(\mathbf{0}, R))$, illustrated in Figure 2 by a light gray region and a dark gray region, respectively.

Given that we approximately describe the process of transmitting APs around AP $\mathbf{0}$ as an inhomogeneous Poisson process of intensity $\lambda_{a}^{0}(x) h(x, \lambda, R)$, we can easily obtain the characteristic function of the cumulative interference adapting (11):

$\psi_{I}(s)=e^{-\int_{0}^{2 \pi} \int_{0}^{r} \lambda_{a}^{0}(b(\rho, \theta)) h(b(\rho, \theta), \lambda, R)\left[1-\phi_{F}(s P l(\rho))\right] \rho \mathrm{d} \rho \mathrm{d} \theta}$

where we remind that the spatial integrals are computed using polar coordinates centered at the receiving node, being $b(\rho, \theta)$ the distance of the generic point $(\rho, \theta)$ from the AP transmitting the useful signal. The success probability $p_{s}(r)$ can finally be obtained plugging $\psi_{I+N}(s)=\psi_{I}(s) e^{-s N_{0}}$ in (11), (12), and (13), respectively for general, Rayleigh or phase type fading distribution.

At last we wish to emphasize that our Matérn model of AP locations can be extended to evaluate also throughput distributions, following the same approach outlined in Section $\mathrm{V}$, which essentially requires to replace $\lambda_{A}$ with $\lambda_{A}^{0}(x)$ and $h\left(z, \lambda_{A}\right)$ with $h(z, \lambda, R)$ in (14) and (15).

\section{Distance distribution between transmitter and receiver}

To compute the throughput according to (4), we also need to evaluate the distribution $f_{D}(r$, Matérn) of the distance $r$ between an AP and its intended receiver, which requires to compute the distance distribution between a node $n$ arbitrarily placed on the plane and the closest node belonging to $\Phi_{A}$ (denoted with $A$ ). When $\Phi_{A}$ is a (hard-core) Matérn point process, the distance distribution is (to the best of our knowledge) not known in closed form. Therefore we propose to approximate it with a form similar to (5), which is sufficiently accurate for our purposes. Our approximation is based on a simple observation, which allows to modify (5) adapting it to the case of a Matern process of parameters $(\lambda, R)$.

The observation is that, for all values $r$ such that $2 r \leq R$, the exact probability $f_{D}(r$, Matérn) is

$$
f_{D}(r, \text { Matérn })=2 \pi r \lambda p \quad 2 r \leq R
$$

where $p=\frac{1-e^{-\lambda \pi R^{2}}}{\lambda \pi R^{2}}$ is the probability of retaining a point in the original Poisson point process. Indeed, consider the case

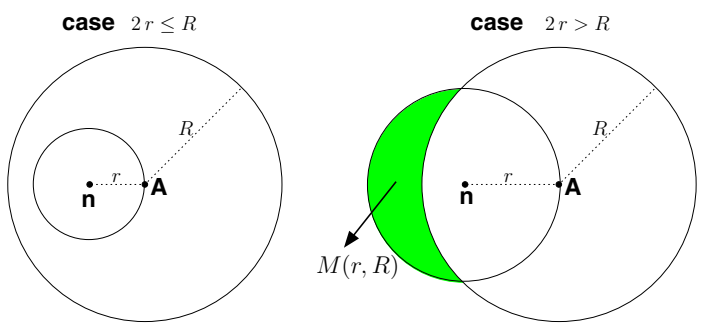

Fig. 3. Illustration of the two cases arising in the approximate computation of the distance distribution between a node $n$ and its closest Access Point $A$, in the case of a Matérn process of APs.

$2 r \leq R$ illustrated in the left part of Figure 3. Once we know that there is a point $A \in \Phi_{A}$ at distance $d \in[r, r+\mathrm{d} r]$ (this event has probability $2 \pi r \lambda p \mathrm{~d} r$ ), we do not need to worry about the presence of other APs closer to $n$ than $A$ (i.e., other nodes belonging to $\Phi_{A}$, lying within the disk of radius $r$ centered at $n$ ). Indeed, when $2 r \leq R$ this disk is entirely contained in the disk of radius $R$ centered at $A$, which by definition cannot contain any other point belonging to $\Phi_{A}$.

Instead, in the case $2 r>R$, we need to worry about the possible presence of APs closer to $n$ than $A$, but only in the area denoted by a shaded region in the right part of Figure 3 . Our approximation is to assume that the existence of these closer APs depends uniquely on the area $M(r, R)$ of the region where we can find them. The value of $M(r, R)$ can be computed in closed form applying elementary geometry, and we omit its expression here. Moreover, we assume that nodes belonging to $\Phi_{A}$ populate area $M(r, R)$ with a probability equivalent to that of a virtual Poisson point process of intensity $\lambda^{*}$. Hence we write

$$
f_{D}(r, \text { Matérn }) \approx 2 \pi r \lambda p e^{-\lambda^{*} M(r, R)}
$$

which is valid also for $2 r \leq R$, assuming that $M(r, R)=0$ in this case. At last, the value of $\lambda^{*}$ to be used in (22) is the only one that guarantees that $f_{D}(r$, Matérn $)$ is a proper pdf, i.e., it is the unique value $\lambda^{*}$ such that $\int_{0}^{\infty} 2 \pi r \lambda p e^{-\lambda^{*} M(r, R)} \mathrm{d} r=1$. We have found that this simple approximation provides sufficiently accurate distributions for all values of $\lambda$ and $R$.

\section{NUMERICAL RESULTS AND INSIGHTS}

In this section we report a selection of the most interesting results that can be obtained following the approach presented in this paper. Since our formulas contain several approximations, model predictions are checked against results obtained by a Montecarlo simulator of the system as described in Section III. The use of a Matérn process to describe the pattern of simultaneous transmitters in CSMA networks has been already discussed in [10], [5].

In all presented cases, we will always assume (due to lack of space) that $P=1, \alpha=3, \beta=1$. We start considering a scenario in which APs are placed according to a Poisson process with intensity $\lambda_{a}=1 / \pi$, while fading is exponentially (Raileigh) distributed with mean $1 / \mu=1$.

Fig. 4 reports the average AP throughput $\mathcal{T}$, the average transmission probability $p_{T}=\int_{0}^{\infty} p_{T}(r) f_{D}(r) \mathrm{d} r$ and the average success probability $p_{s}=\int_{0}^{\infty} p_{s}(r) f_{D}(r) \mathrm{d} r$, as function of the sensing threshold $\sigma$. Models predictions turn out to 


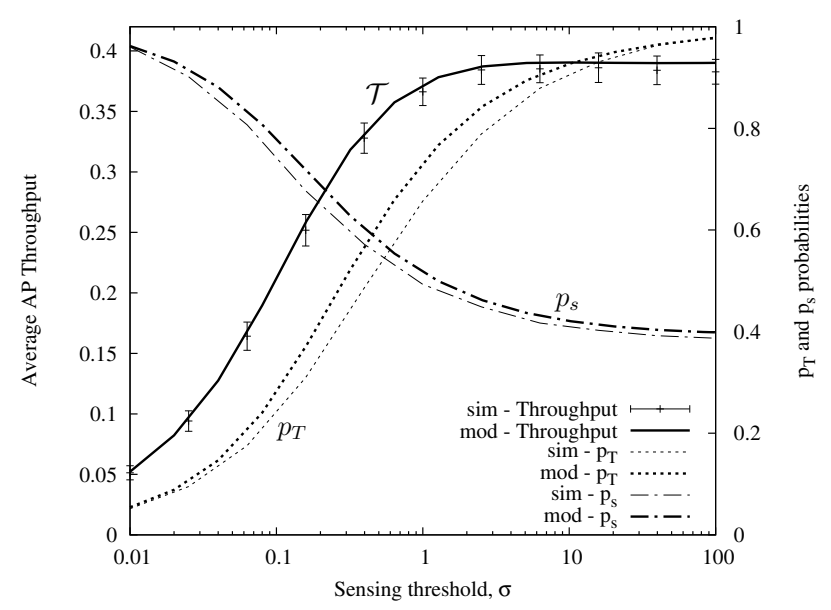

Fig. 4. Average AP throughput (left y axis), average transmission probability $p_{T}$ and average success probability $p_{s}$ (right y axis), as function of the sensing threshold $\sigma$, for $\lambda_{A}=1 / \pi$, Rayleigh fading.

be very accurate, despite the several approximations (for the throughput figure we have also reported $99 \%$-level confidence intervals derived from our simulations).

As expected, the transmission probability $p_{T}$ increases with $\sigma$ (since it is less likely to sense nearby nodes and thus refrain from transmitting), whereas the success probability $p_{s}$ decreases with $\sigma$ (since there is more interference). It is instead less obvious that the overall effect is to produce an AP throughput that increases with $\sigma$ up to a maximum value, after which the throughput decreases very little as we further augment $\sigma$ (a similar behavior occurs for different values of $\alpha$ and $\beta$ ). Recall that we assume that users are always associated to the closest AP (the decay of the throughput after the optimum value can be more pronounced under different user-AP association models). Looking only at Fig. 4 it would be tempting to conclude that in dense random networks the sensing mechanism is not that useful (at least under the closestAP user association), since an aloha-like protocol ${ }^{2}$ would achieve nearly maximum throughput. However, the spatial average of AP throughput provides alone a limited view of the system behavior, and here is exactly where our approach to estimating the throughput distribution comes into play to better understand the role of sensing.

Fig. 5 reports the estimated cumulative distribution function (cdf) of the AP throughput in the same setting of Fig. 5, for three values of $\sigma=0.1,1,10$. We observe that the sensing threshold has a dramatic impact on the throughput distribution. Indeed for large values of $\sigma$ a significant fraction of APs (those in more unfavorable topological conditions) experience negligible throughput, being their transmissions systematically affected by strong interference. Reducing $\sigma$ (i.e., increasing the sensing range) permits to even out the throughput of contending APs in a sensing neighborhood, at the cost of a reduction in the average AP throughput. Although capacity-fairness tradeoffs are commonly found in many communication systems, ours is probably the first analytical model to show such trade-

\footnotetext{
${ }^{2}$ As $\sigma$ tends to infinity, the system behaves like slotted-Aloha with transmit probability equal to 1 .
}
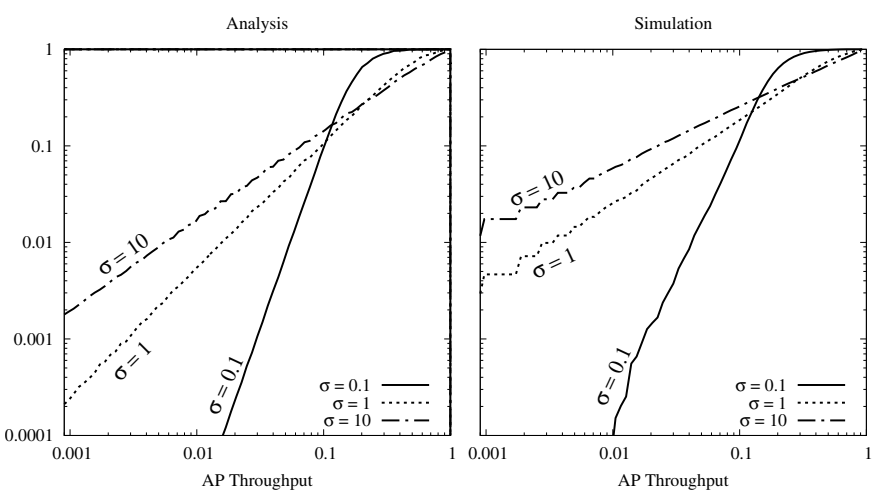

Fig. 5. CDF of AP throughput, for different values of the sensing threshold $\sigma$, in the same scenario as of Figure 4. Comparison between analysis (left plot) and simulation (right plot).

off in the stochastic geometry framework of random CSMA networks. We observe that our approach to estimating the throughput distribution captures most (not all) of the variability measured in simulation, well predicting the impact of $\sigma$.
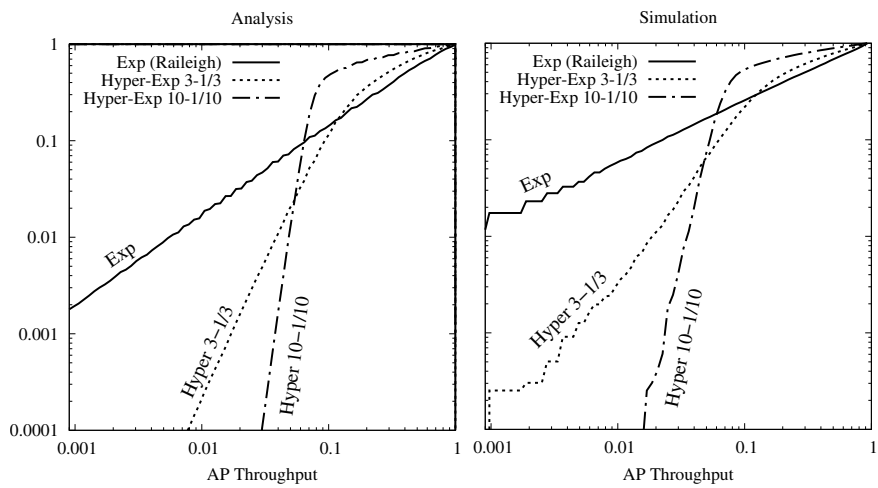

Fig. 6. $\mathrm{CDF}$ of $\mathrm{AP}$ throughput, for different distributions of fading/shadowing, in the case of $\sigma=10, \lambda_{A}=1 / \pi$. Comparison between analysis (left plot) and simulation (right plot).

Another interesting effect that can be captured by our analysis is that related to the shape of the fading/shadowing variable. In Fig. 6 we show again the cdf reported in 5 in the case of $\sigma=10$, which was found to produce a severe unbalance in the throughput distribution under Rayleigh fading. This time, we increase the variation coefficient of the fading/shadowing variable (while maintaining the same mean), considering two second-order hyper-exponential distributions of parameters $\mu_{1}=1 / 3, \mu_{2}=3$ (moderate variation coefficient) or $\mu_{1}=1 / 10, \mu_{2}=10$ (large variation coefficient). We observe that higher diversity in the signal attenuation over the wireless links can help to alleviate the starvation of APs in unfavorable topological conditions, by actually reducing the variability in the throughput distribution (again at the expense of a reduction in the average throughput, not shown here). Again, the model captures fairly well this counter-intuitive phenomenon.

Next, we evaluate the effect of different APs placements on the average throughput and its distribution. Fig. 7 reports the spatial average $\mathcal{T}$ as function of the average density $\bar{\lambda}$ of APs in the network, in the case of Rayleigh fading with 


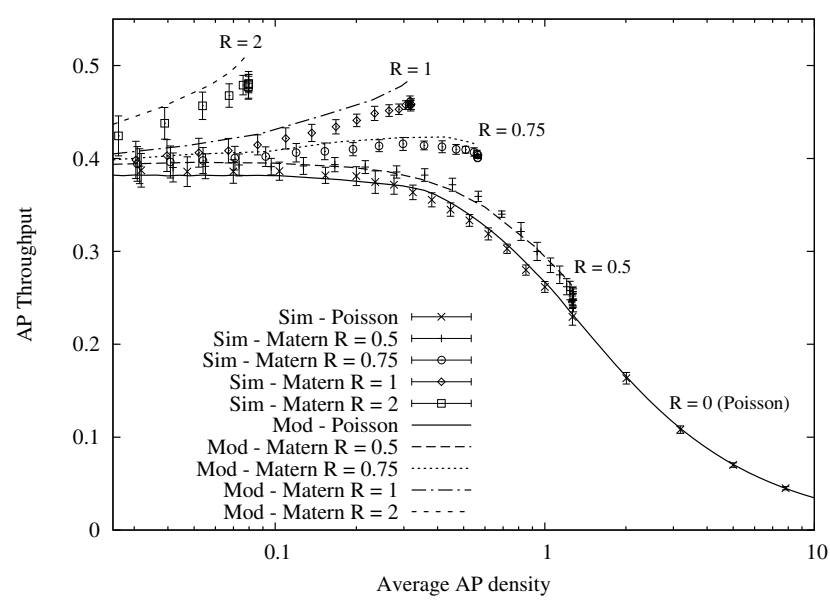

Fig. 7. Average AP throughput as function of the (average) AP density $\lambda_{a}$, for different point processes of APs, in the case of $\sigma=1$, Rayleigh fading.

$\sigma=1$. Besides the Poisson process (for which $\bar{\lambda}=\lambda_{a}$ ), we consider hard-core Matérn processes $(\lambda, R)$ with different radius $R$, in which we let $\lambda$ vary from very small to very large values, obtaining average node densities $\bar{\lambda}=\frac{1-e^{-\lambda \pi R^{2}}}{\pi R^{2}}$. For given $\bar{\lambda}$, we observe that the average AP throughput increases with $R$ (but notice that $R$ cannot exceed $1 / \sqrt{\pi} \bar{\lambda}$ ), which can be explained by the fact that the resulting topologies become more and more regular, with a beneficial effect also on the distance distribution between AP and user. What is somehow surprising is that, for fixed $R$, the AP throughput can even increase for increasing values of the APs density, if $R$ is sufficiently large (see curves related to $R=0.75,1,2$ ). This can be again attributed to the fact that, for a given $R$, Matérn processes become more regular by increasing $\lambda$ (and $\bar{\lambda})$, although this beneficial effect can be offset, for small $R$, by the increased interference. We observe, again, that our model is able to capture fairly well this interesting phenomenon.
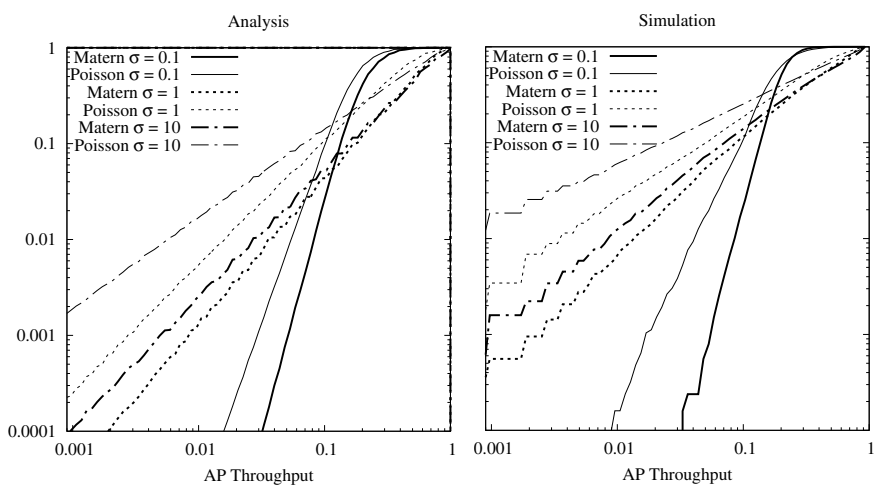

Fig. 8. CDF of AP throughput, for different values of the sensing threshold $\sigma$, in the case of a Matérn process with $R=1, \lambda=10 / \pi$, and the corresponding Poisson (with the same AP density), for Rayleigh fading. Comparison between analysis (left plot) and simulation (right plot).

At last, Fig. 8 reports the throughput cdf for a fixed Matérn process with $\lambda=10 / \pi$ and $R=1$. We consider Rayleigh fading, and different values of the sensing threshold $\sigma=0.1,1,10$. We have also reported the corresponding cdf's resulting from a Poisson process having the same average AP density. We observe that, besides increasing the average AP throughput (see Fig. 7), a more regular displacement of the APs with respect to the Poisson process is also able to reduce the variability in the throughput distribution, as correctly predicted by the model.

\section{CONCLUSIONS}

In this paper we have extended the stochastic geometry approach to modeling dense CSMA networks. So far, spatial averages of performance measures have provided only a limited, possibly misleading view of the system behavior, and therefore they must be supplemented with an analysis of the spatial distribution of the same measures. We have shown how the stochastic geometry analysis can provide fundamental insights into the throughput distribution, especially how the inherent unbalance due to the randomness in the topology is affected by the sensing mechanism and the variability of radio signal propagation. Moreover, we have proposed a methodology that permits to consider scenarios in which APs are not distributed according to a Poisson process, obtaining additional insights that could guide the topology design and control of densely deployed CSMA networks. We believe that our work offers an important contribution in the direction of exploring the potentialities of the stochastic geometry approach to modeling wireless networks. In particular we believe that the methodology devised in this paper can be successfully applied to the design of better performing, and more energy efficient new generation WiFi networks.

\section{REFERENCES}

[1] G. Bianchi, "Performance analysis of the IEEE 802.11 distributed coordination function," IEEE JSAC, vol. 18, pp. 235-247, 2000.

[2] M. Garetto, T. Salonidis, and E.-W. Knightly, "Modeling per-flow throughput and capturing starvation in CSMA multi-hop wireless networks," IEEE/ACM Trans. Netw., vol. 16, no. 4, pp. 864-877, 2008.

[3] R. Boorstyn, A. Kershenbaum, B. Maglaris, and V. Sahin, "Throughput analysis in multihop CSMA packet radio networks," IEEE Transactions on Communications, vol. 35, pp. 267-274, 1987.

[4] M. Durvy, O. Dousse, and P. Thiran, "On the fairness of large CSMA networks," IEEE JSAC, vol. 27, pp. 1093-1104, 2009.

[5] F. Baccelli and B. Błaszczyszyn, "Stochastic geometry and wireless networks, vol. 2: Applications," Foundations and Trends in Networking, vol. 4, no. 1-2, pp. 1-312, 2009.

[6] F. Baccelli and B. Błaszczyszyn, "A New Phase Transition for Local Delays in MANETs," In Proc. IEEE INFOCOM 2010.

[7] M. Haenggi and R. K. Ganti, "Interference in Large Wireless Networks," Foundations and Trends in Networking, Vol. 3, no. 2, pp. 127-248, 2009.

[8] F. Baccelli, B. Błaszczyszyn, and P. Mühlethaler, "An aloha protocol for multihop mobile wireless networks," IEEE Transactions on Information Theory, vol. 52, no. 2, pp. 421-436, 2006.

[9] F. Baccelli, B. Błaszczyszyn, and P. Mühlethaler, "A stochastic model for spatial and opportunistic aloha," IEEE JSAC, vol. 27, no. 7, pp. 1105-1119, 2009.

[10] H. Nguyen, F. Baccelli, and D. Kofman, "A Stochastic Geometry Analysis of Dense IEEE 802.11 Networks," IEEE INFOCOM 2007.

[11] A. Busson and G. Chelius, "Point processes for interference modeling in CSMA/CA ad-hoc networks," PE-WASUN 2009.

[12] I. Palasti, "On some random space filling problem," Publ. Math. Inst. Hung. Acad. Sci., vol. 1, no. 5, pp. 353-359, 1960.

[13] A. Hasan and J. Andrews, "The guard zone in wireless ad hoc networks," IEEE Trans. on Wireless Comm., vol. 6, no. 3, pp. 897-906, 2007.

[14] M. Kaynia, G. E. Øien, and N. Jindal, "Impact of fading on the performance of ALOHA and CSMA," SPAWC 2009.

[15] R. K. Ganti, J. G. Andrews, and M. Haenggi, "High-SIR Transmission Capacity of Wireless Networks with General Fading and Node Distribution," submitted to IEEE Transactions on Information Theory, 2010. 